\title{
High-Density Lipoprotein Cholesterol Level Is Associated With Fibrous Cap Thickness in Acute Coronary Syndrome
}

\author{
Yuichi Ozaki, MD; Atsushi Tanaka, MD, PhD; Kenichi Komukai, MD; \\ Kohei Ishibashi, MD; Takashi Tanimoto, MD, PhD; \\ Hironori Kitabata, MD, PhD; Yasushi Ino, MD; Takashi Kubo, MD, PhD; \\ Toshio Imanishi, MD, PhD; Takashi Akasaka, MD, PhD
}

\begin{abstract}
Background: Although low high-density lipoprotein cholesterol (HDL-C) level has been reported as an independent risk factor for coronary artery disease, few studies addressed the direct relationship between the presence of thin-cap fibroatheroma (TCFA) that is considered as vulnerable plaque in pathology and HDL-C level. The aim of this study was to investigate whether lesion vulnerability is related to HDL-C level in patients with acute coronary syndrome (ACS).

Methods and Results: A total of 261 patients with ACS who underwent optical coherence tomography prior to percutaneous coronary intervention, were enrolled. Patients were divided into a TCFA group $(n=124)$ and a nonTCFA group ( $n=137$ ). TCFA was defined as a lipid plaque (lipid content in $\geq 1$ quadrant) covered with $<70 \mu \mathrm{m}$ thickness fibrous caps. There were no differences in patient characteristics and clinical results between the 2 groups except for HDL-C level, low-density lipoprotein cholesterol (LDL-C) level, and high-sensitive C-reactive protein (hs-CRP) level. On multivariate regression analysis, low HDL-C level ( $\beta$ coefficient: $0.302, P<0.001)$, high LDL-C level ( $\beta$ coefficient: $-0.172, P=0.008)$, hs-CRP level $(\beta$ coefficient: $-0.145, P=0.017)$, and current smoking $(\beta$ coefficient: $-0.124, P=0.028$ ) were identified as independent contributors to fibrous cap thickness.
\end{abstract}

Conclusions: HDL-C is correlated with fibrous cap thickness of the culprit lesion in patients with ACS. HDL-C may be considered as a therapeutic target for plaque stabilization. (Circ J 2013; 77: 2982-2989)

Key Words: Acute coronary syndrome; Fibrous cap thickness; High-density lipoprotein cholesterol; Optical coherence tomography

I has been established that recurrence of cardiovascular events, and coronary artery disease (CAD) death rates are reduced by lipid-lowering therapies with statin. Although aggressive statin therapy, aimed at low-density lipoprotein cholesterol (LDL-C), has significantly reduced major cardiovascular events and mortality, ${ }^{1-7}$ cardiac events remain even though LDL-C levels are adequately controlled. ${ }^{8}$ One reason for this may be the presence of a baseline low high-density lipoprotein cholesterol (HDL-C) level even during intensive treatment with statins. ${ }^{9} \mathrm{HDL}-\mathrm{C}$ has been reported as an independent inverse predictor of cardiovascular disease, ${ }^{10-14}$ and regression of coronary atherosclerosis was correlated with the moderate increase in serum HDL-C level. ${ }^{15}$

Although gray-scale intravascular ultrasound (IVUS) has been used to investigate the relationship between blood lipid profile and coronary tissue characteristics, ${ }^{16-18}$ the current consensus is that IVUS is unable to visualize the details of tissue characterization, ${ }^{19}$ including thin-cap fibroatheroma (TCFA), which is a morphological feature of vulnerable plaque. Therefore, it remains unclear whether serum HDL-C level is associated with culprit lesion vulnerability. The aim of this study was to investigate whether lesion vulnerability assessed on optical coherence tomography (OCT) is related to HDL-C level in patients with acute coronary syndrome (ACS).

\section{Methods}

\section{Subjects}

The subjects consisted of 261 consecutive ACS patients (with or without ST-segment elevation) who were admitted to

Received April 15, 2013; revised manuscript received July 10, 2013; accepted August 1, 2013; released online September 4, 2013 Time for primary review: 21 days

Department of Cardiovascular Medicine, Wakayama Medical University, Wakayama, Japan

Mailing address: Atsushi Tanaka, MD, PhD, Department of Cardiovascular Medicine, Wakayama Medical University, 811-1, Kimiidera, Wakayama 641-8510, Japan. E-mail: a-tanaka@wakayama-med.ac.jp

ISSN-1346-9843 doi:10.1253/circj.CJ-13-0512

All rights are reserved to the Japanese Circulation Society. For permissions, please e-mail: cj@j-circ.or.jp 

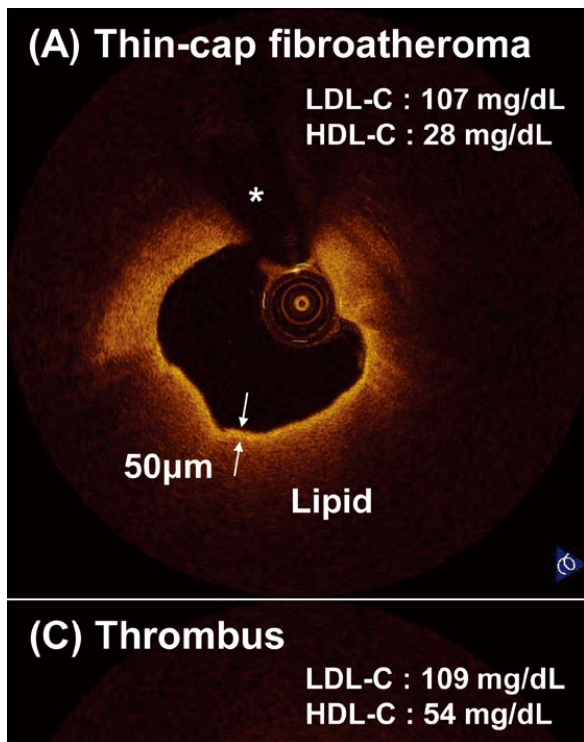

(B) Plaque rupture

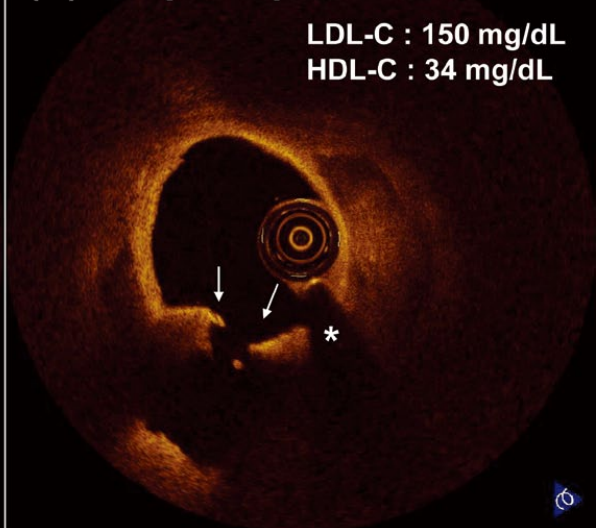

(D) Calcification

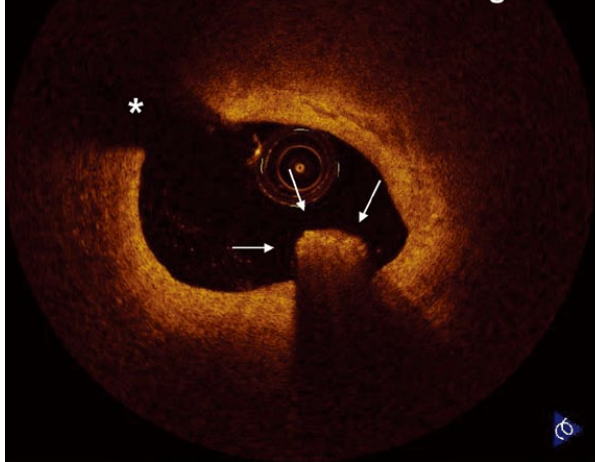

LDL-C : $84 \mathrm{mg} / \mathrm{dL}$

HDL-C : $52 \mathrm{mg} / \mathrm{dL}$

6

Figure 1. Representative cases of (A) thin-cap fibroatheroma, (B) plaque rupture, (C) thrombus, and (D) calcification. (A) The thickness of the fibrous cap is $50 \mu \mathrm{m}$ (arrows). (B) Fibrous cap was broken at the midportion (arrows). (C) Fibrous plaque was observed, and intracoronary thrombus (arrows) protruding into the vessel lumen from the surface of the vessel wall. (D) Calcium deposit showing a sharply delineated region with a signal-poor interior (arrows). HDL-C, high-density lipoprotein cholesterol; LDL$\mathrm{C}$, low-density lipoprotein cholesterol. Asterisk, guidewire artifact.

Wakayama Medical University Hospital (Wakayama, Japan) and underwent OCT prior to percutaneous coronary intervention (PCI). We excluded patients with cardiogenic shock, unstable hemodynamic status, unsuccessful reperfusion of Thrombolysis In Myocardial Infarction (TIMI) grade 3 coronary flow on initial aspiration thrombectomy before OCT imaging, and unwillingness to participate. Patients were divided into a TCFA or a non-TCFA group according to preintervention OCT findings of the culprit lesion.

This study complied with the Declaration of Helsinki with regard to investigation in humans, and the protocol for this study was approved by the Ethics Committee of Wakayama Medical University. We also obtained written informed consent from all participants before coronary angiography (CAG).

\section{Study Protocol}

All patients received oral aspirin $(162 \mathrm{mg})$, an i.v. bolus injection of 5,000 IU of heparin, and i.c. isosorbide dinitrate $(2 \mathrm{mg})$ before CAG. After completion of diagnostic CAG, culprit lesion was identified on the basis of CAG findings as well as those of electrocardiography and echocardiography.

Following CAG, OCT imaging was performed using a 6-F guiding catheter to observe the culprit lesion before any interventions. If the patient had TIMI grade 0,1 , or 2 coronary flow, aspiration thrombectomy was performed using an aspiration catheter (Export ${ }^{\circledR}$; Medtronic Japan, Tokyo, Japan) before OCT image acquisition. Frequency-domain OCT (FD-OCT) or time-domain OCT (TD-OCT) was used for OCT image acquisitions. The FD-OCT system (C7-XR ${ }^{\mathrm{TM}}$ FD-OCT imaging system; LightLab Imaging, Westford, MA, USA) consists of an intravascular OCT catheter (RX ImageWire II; LightLab Imaging), an imaging engine, probe interface unit, and a computer console, which also contains the data acquisition board. For FD-OCT, the 2.7-F imaging catheter can be delivered as a monorail rapid-exchange catheter over a 0.014 -in PCI guidewire through a 6-F guiding catheter and was positioned so that its imaging lens was distal to the culprit lesion. All OCT images from the FD-OCT system were obtained using an automatic pullback device traveling at a rate of $20 \mathrm{~mm} / \mathrm{s}$. For tight lesions, alternatively TD-OCT was used for OCT image acquisition with an automatic pullback device traveling at a rate of $1.0 \mathrm{~mm} / \mathrm{s}$. To flush the vessel, we infused a mixture of commercially available dextran 40 and lactated Ringer's solution (Low Molecular Dextran L Injection ${ }^{\circledR}$; Otsuka Pharmaceuti- 


\begin{tabular}{|c|c|c|c|}
\hline & $\begin{array}{c}\text { TCFA group } \\
(n=124)\end{array}$ & $\begin{array}{c}\text { Non-TCFA group } \\
(n=137)\end{array}$ & P-value \\
\hline Age (years) & $66 \pm 12$ & $67 \pm 10$ & 0.44 \\
\hline Male gender & $96(77)$ & $103(75)$ & 0.67 \\
\hline \multicolumn{4}{|l|}{ Coronary risk factors } \\
\hline Hypertension & $78(63)$ & $94(69)$ & 0.33 \\
\hline Diabetes mellitus & $47(38)$ & $57(42)$ & 0.54 \\
\hline Dyslipidemia & $68(55)$ & $76(56)$ & 0.92 \\
\hline Current smoking & $64(52)$ & $61(45)$ & 0.25 \\
\hline Family history & $21(17)$ & $24(18)$ & 0.90 \\
\hline Obesity & $33(27)$ & $32(23)$ & 0.54 \\
\hline Total cholesterol (ml/dl) & $187.8 \pm 36.4$ & $182.1 \pm 33.9$ & 0.19 \\
\hline Triglyceride (mg/dl) & $118.9 \pm 71.5$ & $104.5 \pm 47.9$ & 0.06 \\
\hline LDL-C (mg/dl) & $120.7 \pm 31.1$ & $110.0 \pm 28.1$ & 0.004 \\
\hline HDL-C (mg/dl) & $39.6 \pm 10.1$ & $46.7 \pm 11.7$ & $<0.001$ \\
\hline LDL-C/HDL-C ratio & $3.2 \pm 1.0$ & $2.5 \pm 0.8$ & $<0.001$ \\
\hline $\mathrm{HbA1c}(\%)$ & $6.1 \pm 1.4$ & $6.0 \pm 1.4$ & 0.76 \\
\hline Peak CK (IU/L) & $495(119-2,071)$ & $400(99-2,016)$ & 0.29 \\
\hline Peak CK-MB (IU/L) & $45(11-226)$ & $28(8-180)$ & 0.14 \\
\hline hs-CRP (mg/dl) & $0.14(0.10-0.27)$ & $0.19(0.10-0.39)$ & 0.003 \\
\hline STEMI & $66(53)$ & $64(47)$ & 0.29 \\
\hline Pre-treatment TIMI flow grade & & & 0.49 \\
\hline 0 & $45(36)$ & $46(34)$ & \\
\hline 1 & $13(11)$ & $14(10)$ & \\
\hline 2 & $39(31)$ & $36(26)$ & \\
\hline 3 & $27(22)$ & $41(30)$ & \\
\hline Post-treatment TIMI flow grade & & & 0.38 \\
\hline 0 & 0 & 0 & \\
\hline 1 & 0 & 0 & \\
\hline 2 & $16(13)$ & $13(9)$ & \\
\hline 3 & $108(87)$ & $124(91)$ & \\
\hline \multicolumn{4}{|l|}{ Medication on admission } \\
\hline ACEI or ARB & $49(40)$ & $53(39)$ & 0.89 \\
\hline$\beta$-blocker & $20(16)$ & $27(20)$ & 0.45 \\
\hline Statin & $42(34)$ & $43(31)$ & 0.67 \\
\hline Insulin & $9(7)$ & $17(12)$ & 0.17 \\
\hline
\end{tabular}

Data given as mean $\pm S D$, median (IQR), or $n(\%)$.

$\mathrm{ACEl}$, angiotensin-converting enzyme inhibitor; ARB, angiotensin II receptor blocker; $\mathrm{CK}$, creatine kinase; HbA1c, hemoglobin A1c; HDL-C, high-density lipoprotein cholesterol; hs-CRP, high sensitivity C-reactive protein; LDL-C, low-density lipoprotein cholesterol; MB, myocardial band; STEMI, ST-elevation myocardial infarction; TCFA, thin-cap fibroatheroma; TIMI, thrombolysis in myocardial infarction.

cal, Tokushima, Japan) via a guiding catheter at a rate of $2.5-4.5 \mathrm{ml} / \mathrm{s}$ using an auto injector pump (Mark V; Medrad, Warrendale, PA, USA) for both methods. ${ }^{20,21}$

A total of 3,000 units unfractionated heparin were given every hour during the procedure to maintain an activated clotting time $>300 \mathrm{~s}$. In addition to initial loading of aspirin $(100 \mathrm{mg})$ and clopidogrel $(300 \mathrm{mg})$ prior to PCI, the following dual antiplatelet therapy was also used: aspirin $(81 \mathrm{mg} /$ day $)$ and clopidogrel ( $75 \mathrm{mg} /$ day) after stent implantation. No glycoprotein IIb/IIIa inhibitors were used in this study because these inhibitors have not been approved in Japan.

\section{Analysis of OCT Images}

The OCT findings were interpreted by 2 experienced OCT observers who were blinded to the angiographic and clinical data. When there was any discordance between the observers, a consensus reading was obtained. The culprit lesion was de- termined on CAG, in conjunction with electrocardiography, echocardiography, and angiography. Then, OCT images were co-registered with angiograms using anatomical landmarks such as side branches, morphological features such as calcifications, and fluoroscopy. After identifying the culprit lesion, cap thickness was measured frame by frame to determine the thinnest site. When ruptured cap showed tapered shape, we measured starting point of the taper for cap thickness. If the starting point of the taper was the thinnest in the plaque, we adopted it as the thinnest fibrous cap thickness. For other cases, we simply adopted the thinnest part as the thinnest cap thickness regardless of the cap interval. The highest number of lipid quadrants was analyzed using the OCT frame containing the thinnest cap. OCT images were analyzed according to Consensus Standards for Acquisition, Measurement, and Reporting of Intravascular Optical Coherence Tomography Studies. ${ }^{22}$ Fibrous cap was identified as a tissue layer that is a signal- 


\begin{tabular}{lccr|}
\hline Table 2. OCT Findings of Culprit Lesion & & & \\
& $\begin{array}{c}\text { TCFA } \\
\text { group }(\mathbf{n}=\mathbf{1 2 4})\end{array}$ & $\begin{array}{c}\text { Non-TCFA } \\
\text { group }(\mathbf{n}=\mathbf{1 3 7})\end{array}$ & P-value \\
Plaque rupture & $84(68)$ & $57(42)$ & $<0.001$ \\
Thrombus & $100(81)$ & $79(58)$ & $<0.001$ \\
Calcification & $70(57)$ & $70(51)$ & 0.386 \\
Intimal vasculature & $68(55)$ & $56(41)$ & 0.024 \\
Fibrous cap thickness $(\mu \mathrm{m})$ & $55.8 \pm 7.0$ & $93.5 \pm 20.0$ & $<0.001$ \\
Lipid arc $\left(^{\circ}\right)$ & $145 \pm 65$ & $104 \pm 53$ & $<0.001$ \\
Lipid plaque & & & $<0.001$ \\
$0^{\circ}$ & 0 & $94(69)$ & \\
$1-90^{\circ}$ & $38(31)$ & $25(18)$ & \\
$91-180^{\circ}$ & $57(46)$ & $14(10)$ & \\
$181-360^{\circ}$ & $29(23)$ & $4(3)$ & 0.242 \\
Lipid length $(\mathrm{mm})$ & $15.7 \pm 2.5$ & $16.1 \pm 1.4$ & \\
\hline
\end{tabular}

Data given as mean \pm SD or $\mathrm{n}(\%)$.

OCT, optical coherence tomography. Other abbreviation as in Table 1.

rich homogenous region overlying a lipid core characterized by a diffusely bordered, signal-poor region on the OCT image. ${ }^{22}$ Lipid was semi-quantified according to the number of involved quadrants on the cross-sectional OCT image. Cap thickness was measured frame by frame and 3 times for each image to determine the thinnest site, and the average was calculated. In this study, TCFA was defined as plaque with lipid content in $\geq 1$ quadrant and the thinnest part of the fibrous cap measuring $<70 \mu \mathrm{m}$ because the physical spatial resolution of any current commercially available OCT systems is $>10 \mu \mathrm{m}$ (Figure 1A).23 Plaque rupture was defined as the presence of fibrous cap discontinuity and a cavity formation in the plaque. ${ }^{24}$ Intracoronary thrombus was identified as a mass attached to the luminal surface or floating within the lumen. Calcification was identified as a signal-poor or heterogeneous region with a sharply delineated border (Figures 1B-D). Intimal vasculature appeared as a signal-poor void that was sharply delineated.

To assess the reproducibility of measurements, all OCT images were analyzed by 2 independent observers, and the plaque characterization and fibrous cap thickness were compared to examine inter-observer variability. To determine intra-observer variability, the images were analyzed again by the same 2 observers at least 2 weeks after the initial measurement, and these 2 measurements were compared.

\section{Clinical and Biochemical Parameters}

The following classical coronary risk factors were assessed: age, sex, hypertension (blood pressure $\geq 140 / 90 \mathrm{mmHg}$, and/or a history of taking antihypertensive medication), diabetes mellitus (fasting plasma glucose $\geq 126 \mathrm{mg} / \mathrm{dl}$, casual plasma glucose $\geq 200 \mathrm{mg} / \mathrm{dl}$, or a diabetic pattern on 75 -g oral glucose tolerance test), dyslipidemia (serum total cholesterol level $\geq 220 \mathrm{mg} / \mathrm{dl}$ ), obesity (body mass index $\geq 25 \mathrm{~kg} / \mathrm{m}^{2}$ ), current smoking, and family history.

Blood was collected in the emergency department. The blood samples were centrifuged, and the serum was removed and stored at $-80^{\circ} \mathrm{C}$ until the assays could be performed. Highsensitivity C-reactive protein (hs-CRP) was analyzed using a commercially available testing kit (N-Latex CRP II; Dade Behring Marburg, Marburg, Germany).

\section{Statistical Analysis}

All statistical analyses were performed using SPSS version 11.0
(SPSS, Chicago, IL, USA). Data are expressed as mean \pm SD deviation for normally distributed variables and median (interquartile range [IQR]) for skewed variables. Categorical data are presented as $\mathrm{n}(\%)$. Differences between 2 groups were tested using unpaired t-test for normally distributed variables; Mann-Whitney test for skewed variables; and chi-squared test or Fisher's exact test as appropriate for categorical variables. Simple linear regression was used for assessing the correlation between 2 parameters. To test the intra- and inter-observer agreement, $\kappa$ was calculated for TCFA and the correlation coefficient was calculated for fibrous cap thickness. In addition, the bias between observers was tested. Multivariate linear regression was used to identify the contributors for fibrous cap thickness and multivariate logistic regression was used to determine the contributors for presence of TCFA. Those variables that had $\mathrm{P}<0.05$ on univariate analysis for the presence of TCFA (HDL-C, LDL-C, and hs-CRP) and clinically meaningful factors for plaque vulnerability (classical coronary risk factors and medication of statin and angiotensin-converting enzyme inhibitor or angiotensin II receptor blocker) were included in multivariate regression analysis. $\mathrm{P}<0.05$ was considered statistically significant.

\section{Results}

\section{Patient Characteristics and Clinical Results}

TCFA at culprit site was observed in 124 (48\%) of the 261 patients. Patient characteristics and clinical results at baseline for both groups are summarized in Table 1. There were no statistically significant differences between the 2 groups except for HDL-C level (TCFA, $39.6 \pm 10.1 \mathrm{mg} / \mathrm{dl}$ vs. non-TCFA, $46.7 \pm 11.7 \mathrm{mg} / \mathrm{dl} ; \mathrm{P}<0.001$ ), LDL-C level (TCFA, $120.7 \pm$ $31.1 \mathrm{mg} / \mathrm{dl}$ vs. non-TCFA, $110.0 \pm 28.1 \mathrm{mg} / \mathrm{dl} ; \mathrm{P}=0.004)$, LDL-C/ HDL-C ratio (TCFA, $3.2 \pm 1.0$ vs. non-TCFA, $2.5 \pm 0.8 ; \mathrm{P}<0.001$ ), and hs-CRP level (TCFA, $0.14 \mathrm{mg} / \mathrm{dl}$, IQR $0.10-0.27 \mathrm{mg} / \mathrm{dl}$ vs. non-TCFA, $0.19 \mathrm{mg} / \mathrm{dl}$, IQR $0.10-0.39 \mathrm{mg} / \mathrm{dl} ; \mathrm{P}=0.003$ ). There was no significant difference in terms of the time from onset to blood sampling between the 2 groups (TCFA, $2.6 \mathrm{~h}$, IQR $1.7-4.3 \mathrm{~h}$ vs. non-TCFA, 2.8 h, IQR 2.0-4.0 h; $\mathrm{P}=0.606$ ), although significant difference was observed in ST-elevation myocardial infarction (STEMI) vs. non-STEMI patients $(2.5 \mathrm{~h}$, IQR $1.9-3.5 \mathrm{~h}$ vs. 3.2 h, IQR 1.8-4.8 h; $\mathrm{P}=0.012$ ). 


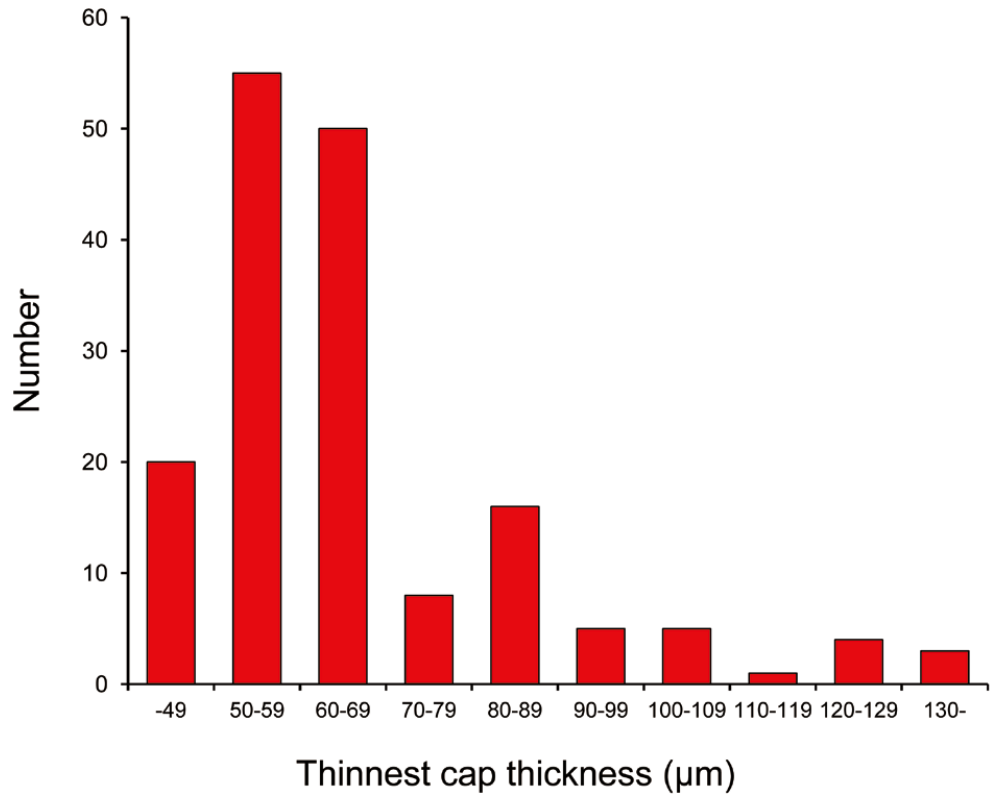

Figure 2. Frequency of thinnest cap thickness.
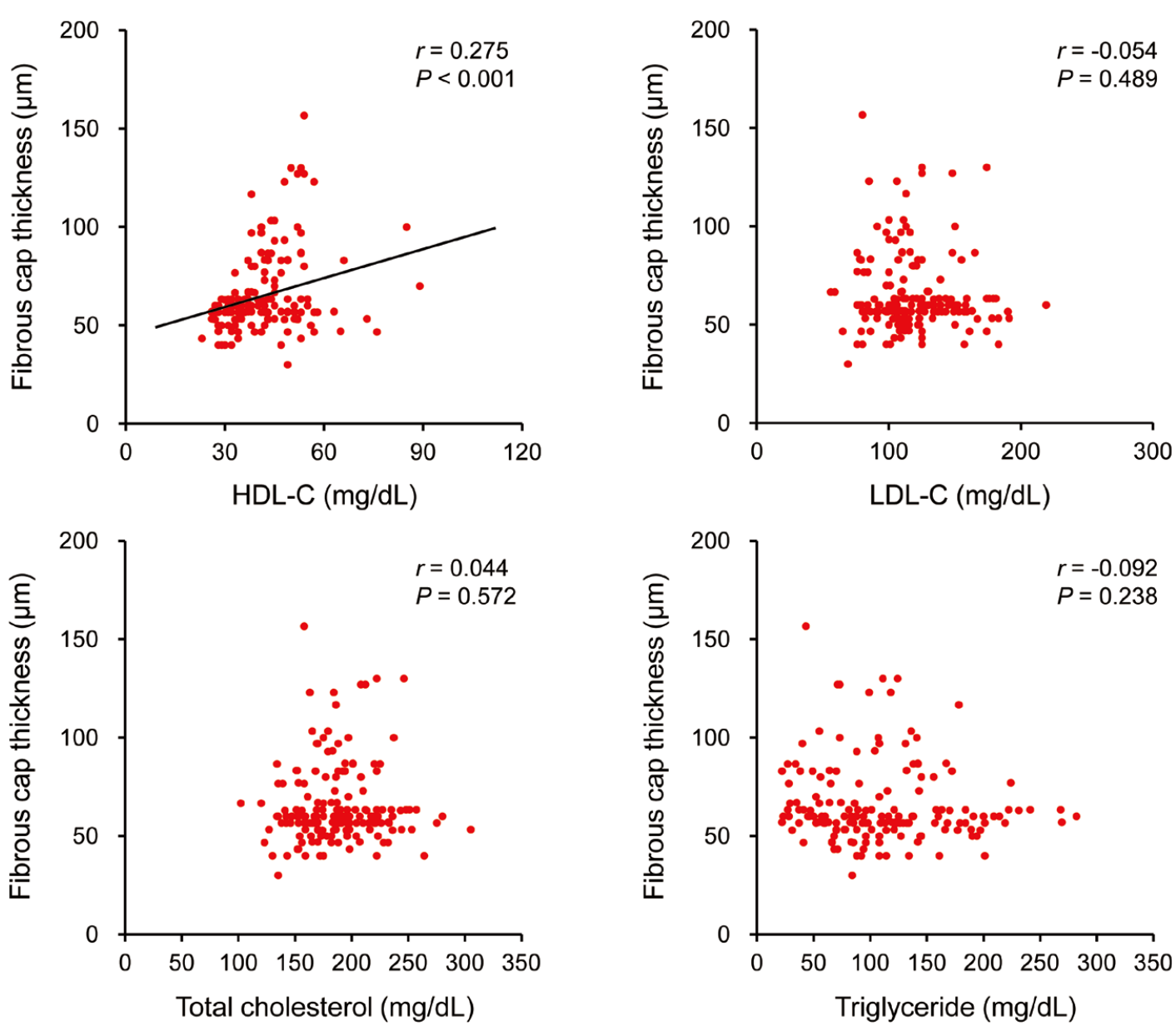

Figure 3. Fibrous cap thickness vs. high-density lipoprotein cholesterol (HDL-C), low-density lipoprotein cholesterol (LDL-C), total cholesterol, and triglyceride level. 


\section{OCT Findings}

Culprit lesions were successfully observed on OCT without any serious complications. The median time from symptom onset to OCT was 4.2h (IQR, 3.3-5.8h). TD-OCT was used in 36 patients (14\%) and FD-OCT in 225 patients. Pre-intervention OCT findings for both groups are summarized in Table 2. Plaque rupture (TCFA, 68\% vs. non-TCFA, 42\%; $\mathrm{P}<0.001$ ), thrombus (TCFA, 81\% vs. non-TCFA, 58\%; $\mathrm{P}<0.001$ ), and intimal vasculature (TCFA, 55\% vs. non-TCFA, 41\%; $\mathrm{P}=$ $0.024)$ at the culprit lesion were significantly more prevalent in patients with TCFA. Lipid plaque was observed in 167 (64\%) patients. Distribution of thinnest cap thickness is given in Figure 2.

The intra- and inter-observer agreements for TCFA were good (intra-observer, $\kappa=0.939$; inter-observer, $\kappa=0.923$, respectively) and the correlation coefficients were high for repeated measurements of fibrous cap thickness by the same observer (bias, $-0.9 \mu \mathrm{m}$; limits of agreement, -17.7 to $+16.1 \mu \mathrm{m}$, $\mathrm{r}=0.923$ ) and by 2 different observers (bias, $-1.2 \mu \mathrm{m}$; limits of agreement, -18.7 to $+16.3 \mu \mathrm{m}, \mathrm{r}=0.916$ ).

\section{Lipoproteins and OCT Findings}

HDL-C level was significantly correlated with fibrous cap thickness $(\mathrm{r}=0.275, \mathrm{P}<0.001)$, but LDL-C, total cholesterol, and triglyceride levels were not (LDL-C, $\mathrm{r}=-0.054, \mathrm{P}=0.489$; total cholesterol, $\mathrm{r}=0.044, \mathrm{P}=0.572$; triglyceride, $\mathrm{r}=-0.092, \mathrm{P}=0.238$, respectively; Figure 3). Moreover, lipid arc was also significantly correlated with HDL-C level $(\mathrm{r}=-0.180, \mathrm{P}=0.020)$, but not with LDL-C level ( $\mathrm{r}=0.065, \mathrm{P}=0.402)$. Lipid length was not associated with both HDL-C level $(\mathrm{r}=-0.030, \mathrm{P}=0.696)$ and LDL-C level ( $r=0.056, \mathrm{P}=0.473)$.

\section{Contributing Factors to TCFA and Fibrous-Cap Thickness}

On multivariate logistic regression model analysis, HDL-C level (odds ratio [OR], 0.930; 95\% confidence interval [CI]: $0.904-0.958 ; \mathrm{P}<0.001)$ and LDL-C level (OR, 1.015; 95\% CI: $1.005-1.025 ; \mathrm{P}=0.002$ ) were independent contributors to TCFA at the culprit lesion (Table 3 ). In patients with lipid plaque, multivariate regression model analysis indicated that HDL-C level ( $\beta$ coefficient, 0.302; $\mathrm{P}<0.001)$, LDL-C level $(\beta$ coefficient, $-0.172 ; \mathrm{P}=0.008)$, hs-CRP level ( $\beta$ coefficient, -0.145 ; $\mathrm{P}=0.017$ ), and current smoking ( $\beta$ coefficient, $-0.124 ; \mathrm{P}=0.028)$ were independent contributors to fibrous cap thickness of the culprit lesion.

When we excluded patients with statin pre-treatment, HDL-C level (OR, 0.933; 95\% CI: 0.901-0.965; $\mathrm{P}<0.001$ ) and LDL-C level (OR, 1.020; 95\% CI: 1.008-1.033; $\mathrm{P}=0.001$ ) were also independent contributors to TCFA at the culprit lesion. In patients assessed using FD-OCT, both HDL-C level and LDL-C level were independent contributors to TCFA at the culprit lesion. Furthermore, HDL-C level, LDL-C level, and hs-CRP level were also independent contributors to fibrous cap thickness of the culprit lesion.

\section{Discussion}

This study has shown that HDL-C is associated with the presence of TCFA and fibrous cap thickness at the culprit lesion. Furthermore, LDL-C, hs-CRP, and smoking were related to plaque vulnerability in patients with ACS.

\section{Lipid Profile and Vulnerable Plaque}

Many clinical trials using statin to lower LDL-C have consistently demonstrated substantial reductions in major cardiovascular events. ${ }^{1-7}$ In spite of the statin therapy, there remains a

\begin{tabular}{|lccc|}
\hline \multicolumn{4}{|c|}{ Table 3. Contributors to TCFA } \\
OR & $95 \%$ Cl & P-value \\
hs-CRP $(\mathrm{mg} / \mathrm{dl})$ & 2.124 & $0.930-4.848$ & 0.074 \\
LDL-C $(\mathrm{mg} / \mathrm{dl})$ & 1.015 & $1.005-1.025$ & 0.002 \\
HDL-C $(\mathrm{mg} / \mathrm{dl})$ & 0.930 & $0.904-0.958$ & $<0.001$ \\
\hline
\end{tabular}

$\mathrm{Cl}$, confidence interval; OR, odds ratio. Other abbreviations as in Table 1.

residual risk in patients with well-controlled LDL-C level. In the Framingham Heart Study, HDL-C level was a more potent risk factor for CAD than LDL-C. ${ }^{13}$ It was also reported that HDL-C level was a predictive factor of major cardiovascular events in patients treated with statin, even when LDL-C level was $<70 \mathrm{mg} / \mathrm{dl} .^{25}$ The "pleiotropic effects" of statins may contribute to plaque stabilization. In the present study, however, when we excluded patients with statin pre-treatment, HDL-C and LDL-C levels were also independent contributors to TCFA at the culprit lesion. It could be possible that statin treatment prevented ACS in some patients via plaque stabilization and thus they were not entered into this study. A different design would be required to evaluate this hypothesis. The present result that HDL-C had an effect on fibrous cap thickening after adjustment for LDL-C could be the missing link between HDL-C level and CAD risk.

Several studies have reported that low HDL-C level is an important risk factor for $\mathrm{CAD},{ }^{26}$ and $\mathrm{HDL}-\mathrm{C}$ has various potentially anti-atherogenic properties, including the mediation of reverse transport of cholesterol from cells of the arterial wall to the liver and steroidogenic organs. ${ }^{27-29}$ Reverse cholesterol transport describes the metabolism and an important antiatherogenic function of HDL-C, namely, HDL-C-mediated efflux of cholesterol from non-hepatic cells and its subsequent delivery to the liver and steroidogenic organs, in which it is used for the synthesis of lipoprotein, bile acids, vitamin D, and steroid hormones. ${ }^{27-29}$ Khera et al reported that the ability of HDL-C to promote cholesterol efflux from macrophage foam cells was strongly and inversely associated with both subclinical atherosclerosis and obstructive CAD. ${ }^{30}$ According to the present results, a treatment strategy solely targeting LDL-C with statin may not be sufficient for CAD management. Therapeutic lipid treatment strategies that involve additional targets beyond LDL-C reduction are needed to improve clinical outcome and it is necessary to pay more attention to modulation of HDL-C similarly to that of LDL-C. Furthermore, it has been reported that low HDL-C is also caused by smoking: ${ }^{31}$ current smokers were less common in the higher HDL-C levels, ${ }^{25}$ and HDL-C and current smoking were independent determinants of plaque rupture. ${ }^{32}$ These data are in accordance with the present results.

\section{Inflammation and Plaque Stability}

Inflammation plays an important role in the development and destabilization of atherosclerotic plaque. ${ }^{33} \mathrm{We}$ have previously reported that broken fibrous cap thickness in ruptured plaque had an inverse correlation with hs-CRP level. ${ }^{24}$ Another study demonstrated that hs-CRP was associated with positive remodeling, fibrous cap thickness, and the presence of TCFA. ${ }^{34,35}$ The current finding that the TCFA group had higher hs-CRP suggests that hs-CRP may also serve as a surrogate marker for plaque stability during HDL-C-targeting lipid therapy. Alwaili et al reported that the ability of HDL-C to promote cholesterol efflux was preserved in ACS, but that the 
HDL-C proteome increased in ACS patients. ${ }^{36}$ Inflammatory proteins in HDL-C from ACS patients might alter the protective effects of HDL-C on the atherosclerosis plaque. Therefore, simple use of HDL-C as a biomarker should be done with caution. Further study is needed in the near future.

\section{Study Limitations}

The present study had a number of limitations. First, the limited penetration depth of OCT does not allow for evaluation of plaque volume. ${ }^{37}$ Therefore, it is difficult to estimate and quantify the amount of lipids on OCT, without spectroscopy. ${ }^{38}$ Second, in the present study, 179 patients $(69 \%)$ had thrombus. Furthermore, among them, $32(12 \%)$ had remaining thrombi that might disturb observation of fibrous cap even after thrombectomy. This intracoronary thrombus at a culprit site may also affect OCT, especially the measurement of fibrous cap thickness, given that thrombus limits the penetration of light or the presence of thrombus itself hinders observation of the microstructure of plaque. Therefore, it is possible that some TCFAs might be misdiagnosed as non-TCFA. Third, we did not perform a 3-vessel assessment, therefore it is possible that some patients might have a vulnerable plaque in another coronary artery as previously reported. ${ }^{39}$ Fourth, the frame level definition of TCFA is deficient because plaque morphology is inherently a 3-D phenomenon. Fifth, the present subjects consisted of patients with 2 different conditions: STEMI and nonSTEMI. The physiopathology of non-STEMI is much less uniform, ranging from a situation similar to STEMI to a mismatch between a severe chronic coronary pathology with severe old lesions and a sudden increase in oxygen demand from the myocardium. In such case, there would be a possibility that OCT might mis-identify the culprit site. Sixth, the number of patients with lipid plaque in the non-TCFA group might have been to small to draw a conclusion. Seventh, discussion would be needed for measurement of broken fibrous cap thickness. Finally, the exclusion of patients without adequate blood flow even after aspiration thrombectomy means that the present results may not be completely representative of all patients with ACS.

\section{Conclusions}

HDL-C is independently correlated with fibrous cap thickness of the culprit lesion in patients with ACS, as well as LDL-C, hs-CRP and current smoking. In addition, the clinical background would be different between patients with and without TCFA. This suggests that various mechanisms are involved in provoking ACS. HDL-C may be considered as a therapeutic target for plaque stabilization.

\section{Acknowledgments}

This work was supported by JSPS KAKENHI Grant Number 24591068.

\section{References}

1. Sever PS, Dahlof B, Poulter NR, Wedel H, Beevers G, Caulfield M, et al. Prevention of coronary and stroke events with atorvastatin in hypertensive patients who have average or lower-than-average cholesterol concentrations, in the Anglo-Scandinavian Cardiac Outcomes Trial--Lipid Lowering Arm (ASCOT-LLA): A multicentre randomised controlled trial. Lancet $2003 ; 361$ : 1149-1158.

2. Sacks FM, Pfeffer MA, Moye LA, Rouleau JL, Rutherford JD, Cole $\mathrm{TG}$, et al. The effect of pravastatin on coronary events after myocardial infarction in patients with average cholesterol levels. Cholesterol and Recurrent Events Trial investigators. N Engl J Med 1996; 335: $1001-1009$.

3. Shepherd J, Cobbe SM, Ford I, Isles CG, Lorimer AR, MacFarlane
PW, et al. Prevention of coronary heart disease with pravastatin in men with hypercholesterolemia. West of Scotland Coronary Prevention Study Group. N Engl J Med 1995; 333: 1301-1307.

4. Downs JR, Clearfield M, Weis S, Whitney E, Shapiro DR, Beere PA, et al. Primary prevention of acute coronary events with lovastatin in men and women with average cholesterol levels: Results of AFCAPS/TexCAPS. Air Force/Texas Coronary Atherosclerosis Prevention Study. JAMA 1998; 279: 1615-1622.

5. Randomised trial of cholesterol lowering in 4444 patients with coronary heart disease: The Scandinavian Simvastatin Survival Study (4S). Lancet 1994; 344: 1383-1389.

6. The Lipid Research Clinics Coronary Primary Prevention Trial results. I. Reduction in incidence of coronary heart disease. JAMA 1984; 251: $351-364$.

7. Baigent C, Keech A, Kearney PM, Blackwell L, Buck G, Pollicino $\mathrm{C}$, et al. Efficacy and safety of cholesterol-lowering treatment: Prospective meta-analysis of data from 90,056 participants in 14 randomised trials of statins. Lancet 2005; 366: 1267-1278.

8. Kones R. Primary prevention of coronary heart disease: Integration of new data, evolving views, revised goals, and role of rosuvastatin in management. A comprehensive survey. Drug Des Devel Ther 2011; 5: 325-380.

9. Sacks FM, Tonkin AM, Shepherd J, Braunwald E, Cobbe S, Hawkins $\mathrm{CM}$, et al. Effect of pravastatin on coronary disease events in subgroups defined by coronary risk factors: The Prospective Pravastatin Pooling Project. Circulation 2000; 102: $1893-1900$.

10. Assmann G, Schulte H, von Eckardstein A, Huang Y. High-density lipoprotein cholesterol as a predictor of coronary heart disease risk. The PROCAM experience and pathophysiological implications for reverse cholesterol transport. Atherosclerosis 1996; 124 (Suppl): $\mathrm{S} 11-\mathrm{S} 20$.

11. Curb JD, Abbott RD, Rodriguez BL, Masaki K, Chen R, Sharp DS, et al. A prospective study of HDL-C and cholesteryl ester transfer protein gene mutations and the risk of coronary heart disease in the elderly. J Lipid Res 2004; 45: 948-953.

12. Sharrett AR, Ballantyne CM, Coady SA, Heiss G, Sorlie PD, Catellier $\mathrm{D}$, et al. Coronary heart disease prediction from lipoprotein cholesterol levels, triglycerides, lipoprotein(a), apolipoproteins A-I and B, and HDL density subfractions: The Atherosclerosis Risk in Communities (ARIC) Study. Circulation 2001; 104: 1108-1113.

13. Gordon T, Castelli WP, Hjortland MC, Kannel WB, Dawber TR. High density lipoprotein as a protective factor against coronary heart disease. The Framingham Study. Am J Med 1977; 62: 707-714.

14. Turner RC, Millns H, Neil HA, Stratton IM, Manley SE, Matthews $\mathrm{DR}$, et al. Risk factors for coronary artery disease in non-insulin dependent diabetes mellitus: United Kingdom Prospective Diabetes Study (UKPDS: 23). BMJ 1998; 316: 823-828.

15. Nicholls SJ, Tuzcu EM, Sipahi I, Grasso AW, Schoenhagen P, Hu $\mathrm{T}$, et al. Statins, high-density lipoprotein cholesterol, and regression of coronary atherosclerosis. JAMA 2007; 297: 499-508.

16. von Birgelen C, Hartmann M, Mintz GS, Baumgart D, Schmermund A, Erbel R. Relation between progression and regression of atherosclerotic left main coronary artery disease and serum cholesterol levels as assessed with serial long-term ( $>$ or $=12$ months) follow-up intravascular ultrasound. Circulation 2003; 108: 2757-2762.

17. Kimura T, Itoh T, Fusazaki T, Matsui H, Sugawara S, Ogino Y, et al. Low-density lipoprotein-cholesterol/high-density lipoprotein-cholesterol ratio predicts lipid-rich coronary plaque in patients with coronary artery disease: Integrated-backscatter intravascular ultrasound study. Circ J 2010; 74: 1392-1398.

18. Kato M, Dote K, Naganuma T, Sasaki S, Ueda K, Okita M, et al. Clinical predictors of culprit plaque rupture assessed on intravascular ultrasound in acute coronary syndrome. Circ J 2010; 74: 1936-1942.

19. Jang IK, Bouma BE, Kang DH, Park SJ, Park SW, Seung KB, et al. Visualization of coronary atherosclerotic plaques in patients using optical coherence tomography: Comparison with intravascular ultrasound. J Am Coll Cardiol 2002; 39: 604-609.

20. Kataiwa H, Tanaka A, Kitabata H, Imanishi T, Akasaka T. Safety and usefulness of non-occlusion image acquisition technique for optical coherence tomography. Circ J 2008; 72: 1536-1537.

21. Ozaki Y, Kitabata H, Tsujioka H, Hosokawa S, Kashiwagi M, Ishibashi $\mathrm{K}$, et al. Comparison of contrast media and low-molecular-weight dextran for frequency-domain optical coherence tomography. Circ J 2012; 76: $922-927$.

22. Tearney GJ, Regar E, Akasaka T, Adriaenssens T, Barlis P, Bezerra $\mathrm{HG}$, et al. Consensus standards for acquisition, measurement, and reporting of intravascular optical coherence tomography studies: A report from the International Working Group for Intravascular Optical Coherence Tomography Standardization and Validation. J Am Coll Cardiol 2012; 59: 1058-1072. 
23. Ozaki Y, Tanaka A, Tanimoto T, Kitabata H, Kashiwagi M, Kubo T, et al. Thin-cap fibroatheroma as high-risk plaque for microvascular obstruction in patients with acute coronary syndrome. Circ Cardiovasc Imaging 2011; 4: 620-627.

24. Tanaka A, Imanishi T, Kitabata H, Kubo T, Takarada S, Tanimoto $\mathrm{T}$, et al. Morphology of exertion-triggered plaque rupture in patients with acute coronary syndrome: An optical coherence tomography study. Circulation 2008; 118: 2368-2373.

25. Barter P, Gotto AM, LaRosa JC, Maroni J, Szarek M, Grundy SM, et al. HDL cholesterol, very low levels of LDL cholesterol, and cardiovascular events. N Engl J Med 2007; 357: $1301-1310$.

26. Gordon DJ, Rifkind BM. High-density lipoprotein: The clinical implications of recent studies. N Engl J Med 1989; 321: 1311-1316.

27. Genest J Jr, Marcil M, Denis M, Yu L. High density lipoproteins in health and in disease. J Investig Med 1999; 47: 31-42.

28. Stein O, Stein Y. Atheroprotective mechanisms of HDL. Atherosclerosis 1999; 144: $285-301$.

29. von Eckardstein A, Assmann G. Prevention of coronary heart disease by raising high-density lipoprotein cholesterol? Curr Opin Lipidol 2000; 11: 627-637.

30. Khera AV, Cuchel M, de la Llera-Moya M, Rodrigues A, Burke MF, Jafri K, et al. Cholesterol efflux capacity, high-density lipoprotein function, and atherosclerosis. N Engl J Med 2011; 364: 127-135.

31. National Cholesterol Education Program Expert Panel on Detection E, Treatment of High Blood Cholesterol in A. Third Report of the National Cholesterol Education Program (NCEP) Expert Panel on Detection, Evaluation, and Treatment of High Blood Cholesterol in Adults (Adult Treatment Panel III) final report. Circulation 2002; 106: 3143-3421.
32. Otsuka F, Hibi K, Kusama I, Endo M, Kosuge M, Iwahashi N, et al. Impact of statin pretreatment on the incidence of plaque rupture in ST-elevation acute myocardial infarction. Atherosclerosis 2010; 213: 505-511.

33. Libby P. Current concepts of the pathogenesis of the acute coronary syndromes. Circulation 2001; 104: 365-372.

34. Kashiwagi M, Tanaka A, Kitabata H, Tsujioka H, Kataiwa H, Komukai $\mathrm{K}$, et al. Feasibility of noninvasive assessment of thin-cap fibroatheroma by multidetector computed tomography. JACC Cardiovasc Imaging 2009; 2: 1412-1419.

35. Kashiwagi M, Tanaka A, Kitabata H, Tsujioka H, Matsumoto H, Arita $\mathrm{Y}$, et al. Relationship between coronary arterial remodeling, fibrous cap thickness and high-sensitivity $\mathrm{C}$-reactive protein levels in patients with acute coronary syndrome. Circ J 2009; 73: 1291-1295.

36. Alwaili K, Bailey D, Awan Z, Bailey SD, Ruel I, Hafiane A, et al. The HDL proteome in acute coronary syndromes shifts to an inflammatory profile. Biochim Biophys Acta 2012; 1821: 405-415.

37. Kubo T, Tanaka A, Kitabata H, Ino Y, Tanimoto T, Akasaka T. Application of optical coherence tomography in percutaneous coronary intervention. Circ J 2012; 76: 2076-2083.

38. Pu J, Mintz GS, Brilakis ES, Banerjee S, Abdel-Karim AR, Maini B, et al. In vivo characterization of coronary plaques: Novel findings from comparing greyscale and virtual histology intravascular ultrasound and near-infrared spectroscopy. Eur Heart J 2012; 33: 372-383.

39. Kato K, Yonetsu T, Kim SJ, Xing L, Lee H, McNulty I, et al. Nonculprit plaques in patients with acute coronary syndromes have more vulnerable features compared with those with non-acute coronary syndromes: A 3-vessel optical coherence tomography study. Circ Cardiovasc Imaging 2012; 5: 433-440. 